\title{
Anti-GD2 hu3F8/Anti-CD3 huOKT3 Bispecific Antibody
}

National Cancer Institute

\section{Source}

National Cancer Institute. Anti-GD2 hu3F8/Anti-CD3 huOKT3 Bispecific Antibody. NCI

Thesaurus. Code C158436.

\begin{abstract}
A bispecific antibody comprised of a humanized anti-CD3 OKT3 (huOKT3) single chain variable fragment (scFv), linked to the carboxyl end of a humanized anti-GD2 3F8 (hu3F8) immunog lobulin G1 (IgG1) light chain, with potential antineoplastic activity. Upon intravenous administration, the anti-GD2 hu3F8/anti-CD3 huOKT3 bispecific antibody binds to CD3 on T-cells and disialog ang lioside GD2 expressed on certain tumor cells, thereby cross-linking T-cells with GD2-expressing tumor cells. This promotes a selective cytotoxic $T$-lymphocyte $(C T L)$ response against GD2-expressing cells. The Fc region of the anti-GD2 hu3F8/anti-CD3 huOKT 3 bispecific antibody has two amino acid substitutions, N297A and K322A, which may prevent cytokine release syndrome and other unwanted side effects including complement-mediated pain. GD2, a disialog ang lioside and tumor-associated antigen (TAA), is overexpressed in a variety of tumor cell types. CD3 is part of the functional T-cell receptor (TCR) complex, which is necessary for antigen recog nition by $T$-cells and is required for signal transduction.
\end{abstract}

